\title{
Psoriasis en el siglo XXI. Perspectivas actuales
}

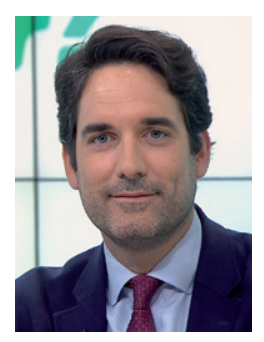

Pablo de la Cueva Dobao Jefe del Servicio de Dermatología. Hospital Universitario Infanta Leonor. Madrid.

\section{INTRODUCCIÓN}

La Organización Mundial de la Salud (OMS), en su informe global, definió la psoriasis como una enfermedad inflamatoria sistémica crónica, incurable e incapacitante, cuya expresión clínica predominante es cutánea. Comporta una gran carga física, emocional y social para los pacientes, cuya calidad de vida puede estar muy afec$\operatorname{tada}^{1}$. La psoriasis suele manifestarse con lesiones cutáneas muy características, si bien, es una enfermedad compleja que puede asociarse a artritis psoriásica y a múltiples enfermedades concomitantes ${ }^{2}$. Los últimos datos de prevalencia en España indican que un 2,3\% de la población padece la enfermedad, sin que exista diferencia entre sexos ${ }^{3}$.

En los últimos años, ha habido grandes avances en el conocimiento de las bases patogénicas, así como en el tratamiento, y esto ha brindado a los pacientes la posibilidad de mejorar sus lesiones cutáneas y su calidad de vida y, así, poder normalizarla. En el presente artículo, se analizará de forma general el estado actual de la enfermedad y los principales retos y necesidades del futuro.

\section{FACTORES ETIOLÓGICOS}

Los factores genéticos son un aspecto fundamental en la patogenia de la psoriasis. En las últimas décadas, ha habido un gran aumento del conocimiento sobre las bases genéticas que intervienen en la susceptibilidad de padecer la enfermedad. La psoriasis no responde a un patrón de herencia monogénica mendeliana, sino a un tipo de herencia poligénica multifactorial: muchos factores genéticos unidos a factores ambientales producen la enfermedad. El principal determinante genético de la psoriasis, el gen 1 de susceptibilidad a la psoriasis (PSORS1; del inglés, psoriasis susceptibility gene 1), se ha localizado en la región cromosómica 6 p21, y se ha determinado que contribuye al 30 $50 \%$ de la susceptibilidad genética de la enfermedad. Se ha confirmado la identificación del alelo HLA-Cw6 en PSORS1 como el principal responsable de la asociación a la psoriasis ${ }^{4,5}$.

En los últimos años, se está analizando la importancia de la variabilidad genética en la repuesta a los tratamientos, pero todavía su aplicabilidad clínica es muy reducida. Se trabaja en la búsqueda de marcadores farmacogenéticos y farmacogenómicos que serían útiles en la predicción de la respuesta clínica, efectuando un tratamiento dirigido más personalizado y, por lo tanto, consiguiendo mejores resultados en salud.

$\mathrm{Al}$ igual que en otras enfermedades inflamatorias inmunomediadas (IMID; del inglés, immune-mediated inflammatory diseases), la psoriasis está 
asociada a una disbiosis en la microbiota intestinal (p. ej., un incremento en Ruminococcus, Blautia, Collinsella, Dorea y Bifidobacterium; y disminución de Bacteroides y Faecalibacterium). Este campo, en gran desarrollo, es otra de las potenciales líneas de investigación de la enfermedad ${ }^{6}$.

\section{ENFERMEDAD SISTÉMICA}

El carácter sistémico de la psoriasis se ha establecido a lo largo de los últimos años, a medida que se ha demostrado la asociación de múltiples enfermedades concomitantes como la artritis psoriásica, el síndrome metabólico, enfermedades cardiovasculares, el hígado graso, la ansiedad y la depresión, entre otras.

Aunque las cifras son muy variables, se considera que aproximadamente el $30 \%$ de los pacientes con psoriasis presenta artritis psoriásica. La detección precoz por parte del dermatólogo y la correcta derivación a reumatología es de suma importancia para evitar daños articulares irreversibles. Recientemente, se han efectuado unas recomendaciones para el cribado de la artritis psoriásica en las consultas de dermatología, que ha de hacerse cada 6-12 meses, preguntando por los siguientes dominios: dolor inflamatorio o nocturno en el esqueleto axial; dolor inflamatorio o tumefacción articular periférica; entesitis (fundamentalmente, en el tendón de Aquiles o las fascias plantares); dactilitis («dedo en salchicha») ${ }^{7}$. La presencia de un hallazgo condiciona una sospecha diagnóstica y la remisión al reumatólogo. Existen encuestas de cribado de artritis psoriásica como el cuestionario de cribado y evaluación de la artritis psoriásica (PASE; del inglés, Psoriatic Arthritis Screening and Evaluation), el instrumento de cribado epidemiológico de la psoriasis (PEST; del inglés, Psoriasis Epidemiology Screening Tool), el cuestionario de cribado de la artritis psoriásica de Toronto (TOPAS; del inglés, Toronto Psoriatic Arthritis Screening Questionnaire), el cuestionario de detección de la artritis temprana en pacientes con psoriasis (EARP; del inglés, Early Arthritis for Psoriatic Patients) y, recientemente, el cuestionario de cribado de la artritis psoriásica de 4 ítems (PURE4; del inglés, 4-item Psoriatic arthritis UnclutteRed screening Evaluation), que todavía no se utilizan de forma mayoritaria, si bien, el PURE4, por su rapidez, puede tener una mayor implantación en un futuro.

En los últimos años, se han desarrollado modelos de colaboración conjunta entre dermatología y reumatología, consiguiendo niveles de satisfacción altos para los pacientes y los propios profesionales, y una mejora de los resultados en salud obtenidos ${ }^{8}$.

Los pacientes con psoriasis - especialmente, las formas graves - presentan un riesgo aumentado de eventos cardiovasculares y mortalidad ${ }^{9}$. En los últimos años, se ha incluido la psoriasis, junto a otras enfermedades inflamatorias, como factor que aumenta el riesgo cardiovascular en las escalas de las diferentes guías. Zarco et al. establecieron unas recomendaciones prácticas para el manejo de la comorbilidad cardiovascular que pasan por la identificación/evaluación de la hipertensión arterial, la dislipidemia, la diabetes mellitus, la obesidad y los hábitos tóxicos, y un manejo secuencial de es$\operatorname{tos}^{10,11}$.

Se está avanzando en las técnicas diagnósticas para efectuar la evaluación del riesgo cardiovascular (p. ej., ecografía femoral, tomografía por emisión de positrones [PET; del inglés, positron emission tomography] con ${ }^{18} \mathrm{~F}$-fluorodesoxiglucosa, etc.), así como para valorar la influencia de los diferentes tratamientos disponibles en el riesgo cardiovascular. Los estudios retrospectivos indican que determinados tratamientos biológicos dirigidos frente a citocinas involucradas en la patogenia de la psoriasis podrían reducir la probabilidad subyacente de desarrollar eventos cardiovasculares. Tanto con los fármacos inhibidores del factor de necrosis tumoral (anti-TNF; del inglés, anti-tumor necrosis factor) como con ustekinumab, existen datos de reducción de la inflamación vascular y un posible efecto cardioprotector. En la actualidad, se están desarrollando estudios retrospectivos y prospectivos con inhibidores de las interleucinas IL-23 e IL-17. 
La frecuencia de hígado graso no alcohólico está aumentada en los pacientes con psoriasis, y es un hallazgo ligado de forma estrecha a la presencia de sobrepeso y obesidad. Su evolución a esteatohepatitis y fibrosis hace que sea necesaria una evaluación de dicho riesgo mediante la realización de estudios bioquímicos — determinación de la aspartato-aminotransferasa (AST o GOT; glutamato-oxalacetato-transaminasa), la alanina-aminotransferasa (ALT o GPT; glutamato-piruvato-transaminasa) y la $\gamma$-glutamiltransferasa (GGT) índices serológicos de fibrosis - p. ej., el índice fibrosis-4 para la fibrosis hepática (FIB-4; del inglés, Fibrosis-4 Index for Liver Fibrosis), el índice del cociente AST/plaquetas (APRI; del inglés, AST to Platelet Ratio Index), o el índice de fibrosis por enfermedad del hígado graso no alcohólico (NFS; del inglés, NAFLD [Non-Alcoholic Fatty Liver Disease] Fibrosis Score)—, o bien técnicas de imagen como la ecografía o la elastografía de transición (FibroScan), según los protocolos establecidos y en colaboración con los hepatólogos ${ }^{12}$. Cobra especial importancia en tratamientos potencialmente hepatotóxicos como el metotrexato.

Otras enfermedades concomitantes emergentes en los últimos años son la enfermedad renal, la periodontitis, la apnea del sueño, la disfunción sexual o la osteoporosis, así como otras IMID como la enfermedad inflamatoria intestinal, la uveítis o la hidradenitis supurativa, y enfermedades autoinmunitarias como la enfermedad celíaca, la alopecia areata, el vitíligo, las enfermedades tiroideas o la cirrosis biliar, y otros trastornos.

Un reciente estudio del Registro Español de tratamientos sistémicos en psoriasis (BIOBADADERM) reflejó que la tasa de fecundidad de las mujeres en edad fértil con psoriasis moderada o grave era un $50 \%$ menor que en la población general ${ }^{13}$. Esto traduce, entre otros, un problema de comunicación en las consultas, que debe mejorarse.

\section{TRATAMIENTO TÓPICO}

El tratamiento tópico sigue siendo el pilar terapéutico de las formas leves, que suponen la mayor parte de los pacientes. Se basa en el empleo de corticoides tópicos y análogos de la vitamina $\mathrm{D}$, así como inhibidores de la calcineurina como el tacrólimus y el pimecrólimus (afectación facial, de pliegues y genital, fuera de indicación), el tazaroteno (derivado de la vitamina $\mathrm{A}$ ), los alquitranes, el ditranol y el ácido salicílico. Se utiliza mucho la combinación de dipropionato de betametasona y un derivado de la vitamina D como el calcipotriol, y su eficacia es mayor en su forma galénica en espuma corporal. Como novedad, se ha estudiado el empleo proactivo dos veces a la semana durante 52 semanas como terapia de mantenimiento, con el objetivo de disminuir los brotes ${ }^{14}$. Uno de los mayores problemas del tratamiento tópico es la falta de adherencia, que hay que tratar de detectar y reducir ${ }^{15}$.

Como tratamientos en fase de desarrollo prometedores, podemos incluir el roflumilast, inhibidor selectivo de la fosfodiesterasa 4 (PDE-4; del inglés, phosphodiesterase type 4), y el tapinarof, activador de los receptores de hidrocarburos de arilos.

\section{TRATAMIENTO SISTÉMICO}

Tanto la fototerapia - en especial, la fototerapia con radiación ultravioleta $\mathrm{B}$ (UVB) de banda estrecha- como los tratamientos sistémicos clásicos tienen vigencia y son ampliamente empleados en la actualidad (metotrexato, acitretina, ciclosporina y fumaratos), a pesar del gran desarrollo de la innovación con los tratamientos biológicos y las moléculas orales.

La investigación de las vías inmunitarias de la psoriasis y el desarrollo de fármacos biológicos que inhiben de forma selectiva pasos clave de la cascada inmunitaria han posibilitado que dispongamos de un amplio arsenal terapéutico, con unos datos de eficacia y seguridad muy elevados. En la actualidad, la Agencia Europea de Medicamentos (EMA; del inglés, European Medicines Agency) ha aprobado los siguientes agentes biológicos para el tratamiento de la psoriasis moderada o grave: infliximab, etanercept, adalimumab, certolizumab, ustekinumab, secukinumab, ixekizumab, brodalumab, guselkumab, tildrakizumab y risankizumab. 
Sus mecanismos de acción, estructura molecular, vía de administración y régimen posológico se detallan en la tabla 1.

La elección del fármaco ha de ser individualizada, considerando factores como la presencia de enfermedades concomitantes, de artritis psoriásica, la edad, el peso, las expectativas y el estilo de vida del paciente, y el riesgo de efectos adversos. Se deben tener en cuenta las características de la enfermedad desde el punto de vista histórico (tratamientos previos) y el grado de actividad de la psoriasis en el momento de la prescripción ${ }^{16}$.

Tabla 1. Propiedades farmacológicas de los agentes biológicos aprobados por la Agencia Europea de Medicamentos para el tratamiento de la psoriasis moderada y grave

\begin{tabular}{|c|c|c|c|c|}
\hline $\begin{array}{l}\text { Tratamiento } \\
\text { aprobado }\end{array}$ & Clase & $\begin{array}{l}\text { Estructura } \\
\text { molecular }\end{array}$ & $\begin{array}{c}\text { Vía de } \\
\text { administración }\end{array}$ & Posología \\
\hline Infliximab & $\begin{array}{l}\text { Inhibidor } \\
\text { del TNF- } \alpha\end{array}$ & $\begin{array}{l}\text { Anticuerpo } \\
\text { monoclonal } \\
\text { quimérico }\end{array}$ & Intravenosa & $\begin{array}{l}5 \mathrm{mg} / \mathrm{kg} \text { las semanas } 0,2 \text { y } 6 . \\
\text { Posteriormente, cada } 8 \text { semanas. }\end{array}$ \\
\hline Etanercept & $\begin{array}{l}\text { Inhibidor } \\
\text { del TNF- } \alpha\end{array}$ & $\begin{array}{l}\text { Proteína } \\
\text { de fusión }\end{array}$ & Subcutánea & $\begin{array}{l}50 \text { mg } 2 \text { veces/semana, } \\
\text { durante } 12 \text { semanas. } \\
\text { Posteriormente, } 1 \text { vez/semana. }\end{array}$ \\
\hline Adalimumab & $\begin{array}{l}\text { Inhibidor } \\
\text { del TNF- } \alpha\end{array}$ & $\begin{array}{l}\text { Anticuerpo } \\
\text { monoclonal } \\
\text { humanizado }\end{array}$ & Subcutánea & $\begin{array}{l}40 \text { mg } \times 2 \text { la semana } 0 \\
40 \text { mg la semana } 1 \\
\text { Posteriormente, } 40 \text { mg cada } \\
2 \text { semanas. }\end{array}$ \\
\hline $\begin{array}{l}\text { Certolizumab } \\
\text { pegol }\end{array}$ & $\begin{array}{l}\text { Inhibidor } \\
\text { del TNF- } \alpha\end{array}$ & $\begin{array}{l}\text { Fragmento Fab' } \\
\text { de un anticuerpo } \\
\text { humanizado } \\
\text { recombinante }\end{array}$ & Subcutánea & $\begin{array}{l}400 \text { mg las semanas } 0,2 \text { y } 4 . \\
\text { Posteriormente, } 200 \text { mg cada } \\
\quad 2 \text { semanas. }\end{array}$ \\
\hline Ustekinumab & $\begin{array}{l}\text { Inhibidor de } \\
\text { las } \mathrm{IL}-12 / 23\end{array}$ & $\begin{array}{l}\text { Anticuerpo } \\
\text { monoclonal } \\
\text { humanizado }\end{array}$ & Subcutánea & $\begin{array}{l}45 \text { o } 90 \mathrm{mg}(>100 \mathrm{~kg}) \\
\text { las semanas } 0 \text { y } 4 . \\
\text { Posteriormente, cada } 12 \text { semanas. }\end{array}$ \\
\hline Secukinumab & $\begin{array}{l}\text { Inhibidor } \\
\text { de la IL-17A }\end{array}$ & $\begin{array}{l}\text { Anticuerpo } \\
\text { monoclonal } \\
\text { humanizado }\end{array}$ & Subcutánea & $\begin{array}{l}300 \mathrm{mg} \text { las semanas } 0,1,2,3 \text { y } 4 \text {. } \\
\text { Posteriormente, cada } 4 \text { semanas. }\end{array}$ \\
\hline Ixekizumab & $\begin{array}{l}\text { Inhibidor } \\
\text { de la IL-17A }\end{array}$ & $\begin{array}{l}\text { Anticuerpo } \\
\text { monoclonal } \\
\text { humanizado }\end{array}$ & Subcutánea & $\begin{array}{l}160 \mathrm{mg} \text { la semana } 0 ; 80 \mathrm{mg} \\
\text { las semanas } 2,4,6,8 \text { y } 12 \text {. } \\
\text { Posteriormente, cada } 4 \text { semanas. }\end{array}$ \\
\hline Brodalumab & $\begin{array}{l}\text { Inhibidor } \\
\text { del receptor } \\
\text { de la IL-17 }\end{array}$ & $\begin{array}{l}\text { Anticuerpo } \\
\text { monoclonal } \\
\text { humanizado }\end{array}$ & Subcutánea & $\begin{array}{l}210 \text { mg las semanas } 0,1 \text { y } 2 \text {. } \\
\text { Posteriormente, cada } 2 \text { semanas. }\end{array}$ \\
\hline Guselkumab & $\begin{array}{l}\text { Inhibidor } \\
\text { de la IL-23 }\end{array}$ & $\begin{array}{l}\text { Anticuerpo } \\
\text { monoclonal } \\
\text { humanizado }\end{array}$ & Subcutánea & $\begin{array}{l}100 \text { mg las semanas } 0 \text { y } 4 \text {. } \\
\text { Posteriormente, cada } 8 \text { semanas. }\end{array}$ \\
\hline Tildrakizumab & $\begin{array}{l}\text { Inhibidor } \\
\text { de la IL-23 }\end{array}$ & $\begin{array}{l}\text { Anticuerpo } \\
\text { monoclonal } \\
\text { humanizado }\end{array}$ & Subcutánea & $\begin{array}{l}100 \text { mg las semanas } 0 \text { y } 4 \text {. } \\
\text { Posteriormente, cada } 12 \text { semanas. }\end{array}$ \\
\hline Risankizumab & $\begin{array}{l}\text { Inhibidor } \\
\text { de la IL-23 }\end{array}$ & $\begin{array}{l}\text { Anticuerpo } \\
\text { monoclonal } \\
\text { humanizado }\end{array}$ & Subcutánea & $\begin{array}{l}150 \text { mg las semanas } 0 \text { y } 4 \text {. } \\
\text { Posteriormente, cada } 12 \text { semanas. }\end{array}$ \\
\hline
\end{tabular}

IL: interleucina; TNF- $\alpha$ : factor de necrosis tumoral alfa (tumor necrosis factor alpha). 
El objetivo terapéutico al inicio de la era biológica se basaba en la obtención de una mejora en el índice de intensidad y extensión de la psoriasis (PASI; del inglés, Psoriasis Area and Severity Index) del $50 \%$ (PASI 50) o del $75 \%$ (PASI 75), si bien, con el desarrollo de nuevas moléculas más eficaces (inhibidores de la IL-17 y de la IL-23), ha sido posible plantearse objetivos cada vez más «ambiciosos» como una mejora del PASI del 90 o el $100 \%$ (PASI 90/100), una puntuación en el índice de calidad de vida dermatológica (DQLI; del inglés, Dermatology Life Quality Index) de 0 o 1 (DLQI 0/1), o una puntuación en la escala de evaluación global del médico (PGA; del inglés, Physician's Global Assessment) de 0 o 1 (PGA $0 / 1)^{17}$. Recientemente, el concepto de PASI absoluto ha adquirido una importancia creciente, al ajustarse más a la práctica clínica habitual y se estableció como objetivo terapéutico alcanzar un PASI $\leq 2-3^{18}$.

En los últimos años, se dispone en el mercado de fármacos biosimilares (etanercept, infliximab y adalimumab), que suponen un potencial ahorro, al ofrecer un precio menor, lo que es un factor de importancia en la búsqueda de la sostenibilidad de los sistemas sanitarios y la implementación de intervenciones eficientes ${ }^{19}$.

Como fármacos innovadores en fase de desarrollo más avanzada, se encuentran bimekizumab (inhibidor dual de la IL-17A y F) y deucravacitinib, inhibidor oral de la tirosina-cinasa 2 (TYK2; del inglés, tyrosine kinase 2).
Algunos campos específicos de estudio en la actualidad (que exceden los objetivos del presente artículo) son las poblaciones especiales (población pediátrica, embarazadas, ancianos y pacientes frágiles); las localizaciones especiales (cuero cabelludo, pliegues, genital, ungueal); así como las formas graves de difícil tratamiento como la psoriasis pustulosa o la psoriasis eritrodérmica.

\section{NECESIDADES GLOBALES DE LOS PACIENTES CON PSORIASIS}

A pesar del desarrollo de los avances de los últimos años, los datos de las encuestas realizadas a 1265 pacientes por la asociación de pacientes Acción Psoriasis (Proyecto NEXT, año 2019) muestran un grado de insatisfacción importante de los pacientes, de ausencia de tratamiento y de falta de equidad en el acceso a las prestaciones sanitarias.

A fin de mejorar la calidad de vida y la asistencia, la OMS estableció unas recomendaciones sobre el manejo global de la psoriasis (tabla 2).

En esta línea, un proyecto español con la participación de los pacientes denominado «Por una Atención Sanitaria más Humanizada de las Personas con Psoriasis», de la Fundación Humans, insta a la creación de unidades interdisciplinarias de psoriasis (integradas por especialistas en dermatología, reumatología, psicología, farmacia, enfermería, nutrición, endocrinología, trabajo social y atención primaria), un mejor acceso a la asistencia psicológica, la creación de alertas sobre la falta de

Tabla 2. Recomendaciones de la Organización Mundial de la Salud sobre la psoriasis

Llevar a cabo el control de la psoriasis y la prevención de las complicaciones.

Conseguir el acceso universal a los servicios de salud y los medicamentos esenciales.

Tener en consideración las necesidades de los pacientes tanto a nivel asistencial como de tratamiento.

Implantar un modelo asistencial centrado en las personas.

Mejorar las capacidades de los profesionales sociosanitarios.

Empoderar y capacitar a los pacientes para gestionar su enfermedad.

Combatir el estigma y la discriminación. 
adherencia, el acceso a información rigurosa y con leguaje cercano, así como la toma de decisiones compartidas, entre otras mejoras para los pacientes.

En los próximos años, uno de los retos importantes es la adaptación de la innovación tecnológica a los procesos asistenciales, como apoyo al diagnóstico y el tratamiento y la educación en salud de los pacientes.

\section{BIBLIOGRAFÍA}

1. World Health Organization (WHO). Global report on psoriasis. Ginebra: WHO; 2016.

2. Mrowietz U, Steinz K, Gerdes S. Psoriasis: to treat or to manage? Exp Dermatol. 2014;23(10):705-9.

3. Ferrándiz C, Carrascosa JM, Toro M. Prevalencia de la psoriasis en España en la era de los agentes biológicos. Actas Dermosifiliogr. 2014;105(5):504-9.

4. Puig $L$, Julià $A$, Marsal $S$. The pathogenesis and genetics of psoriasis. Actas Dermosifiliogr. 2014;105(6):535-45.

5. Puig L. 100 Preguntas clave en... Psoriasis. Barcelona: Permanyer; 2020.

6. Hidalgo-Cantabrana C, Gómez J, Delgado S, Requena-López S, Queiro-Silva R, Margolles A, et al. Gut microbiota dysbiosis in a cohort of patients with psoriasis. Br J Dermatol. 2019; 181(6):1287-95.

7. Belinchón I, Salgado-Boquete L, López-Ferrer A, Ferran M, Coto-Segura P, Rivera R, et al. Dermatologists' role in the early diagnosis of psoriatic arthritis: expert recommendations. Actas Dermosifiliogr (Engl Ed). 2020;111(10):835-46.

8. Queiro R, Coto $P$, Joven B, Rivera R, Navío Marco T, De la Cueva $P$, et al. Current status of multidisciplinary care in psoriatic arthritis in Spain: NEXUS 2.0 project. Reumatol Clin (Engl Ed). 2020;16(1):24-31.

9. Polachek A, Touma Z, Anderson M, Eder L. Risk of cardiovascular morbidity in patients with psoriatic arthritis: a metaanalysis of observational studies. Arthritis Care Res (Hoboken). 2017;69(1):67-74.

10. Zarco Montejo P, Almodóvar González R, De Higes-Martínez E, Gorgojo-Martínez JJ, Guijarro Herraiz C, López Navas MJ, et al. Delphi-based recommendations for the management of cardiovascular comorbidities in patients with psoriatic arthritis and moderate-to-severe psoriasis. Rheumatol Int. 2020;40(6):969-81.
11. Elmets CA, Leonardi CL, Davis DMR, Gelfand JM, Lichten J, Mehta NN, et al. Joint AAD-NPF guidelines of care for the management and treatment of psoriasis with awareness and attention to comorbidities. J Am Acad Dermatol. 2019;80(4): 1073-113.

12. Olveira A, Herranz P, Montes ML. Psoriasis and fatty liver: a harmful synergy. Rev Esp Enferm Dig. 2019;111(4):314-9.

13. González-Cantero A, Carretero G, Rivera R, Ferrándiz C, Daudén E, De la Cueva P, et al.; BIOBADADERM Study Group. Women with moderate-to-severe psoriasis in Spain (BIOBADADERM registry) show more than a $50 \%$ reduction in ageadjusted fertility rate when compared with the general population. Br J Dermatol. 2019;181(5):1085-7.

14. Lebwohl M, Kircik L, Lacour JP, Liljedahl M, Lynde C, Mørch $\mathrm{MH}$, et al. Twice-weekly topical calcipotriene/betamethasone dipropionate foam as proactive management of plaque psoriasis increases time in remission and is well tolerated over 52 weeks (PSO-LONG trial). J Am Acad Dermatol. 2021;84(5): 1269-77.

15. Puig L, Carrascosa JM, Belinchón I, Fernández-Redondo V, Carretero G, Ruiz-Carrascosa JC, et al.; Panel de Expertos del Consenso Delphi sobre Tratamiento tópico de la psoriasis; Grupo de Psoriasis de la Academia Española de Dermatología y Venereología. Adherence and patient satisfaction with topical treatment in psoriasis, and the use, and organoleptic properties of such treatments: a Delphi study with an expert panel and members of the Psoriasis Group of the Spanish Academy of Dermatology and Venereology. Actas Dermosifiliogr. 2013;104(6):488-96.

16. Puig L, Carrascosa JM, Carretero G, De la Cueva P, LafuenteUrrez RF, Belinchón I, et al.; Spanish Psoriasis Group of the Spanish Academy of Dermatology and Venereology. Spanish evidence-based guidelines on the treatment of psoriasis with biologic agents, 2013. Part 1: on efficacy and choice of treatment. Spanish Psoriasis Group of the Spanish Academy of Dermatology and Venereology. Actas Dermosifiliogr. 2013; 104(8):694-709.

17. Carretero G, Puig L, Carrascosa JM, Ferrándiz L, Ruiz-Villaverde R, De la Cueva P, et al.; Spanish Group of Psoriasis. Redefining the therapeutic objective in psoriatic patients candidates for biological therapy. J Dermatolog Treat. 2018;29(4):334-46.

18. López-Estebaranz JL, De la Cueva-Dobao P, de la Torre Fraga C, Galán Gutiérrez M, González Guerra E, Mollet Sánchez J, et al. Manejo de la psoriasis moderada-grave en condiciones de práctica habitual en el ámbito hospitalario español. Actas Dermosifiliogr. 2018;109(7):631-42.

19. Puig L, López-Ferrer A. Biosimilars for the treatment of psoriasis. Expert Opin Biol Ther. 2019;19(10):993-1000. 